Special Issue of Current Opinion in Physiology: Sex Differences in Physiology

\title{
Editorial Overview:
}

\section{Daylighting Sex Differences in Physiology}

Stephen F. Goodwin ${ }^{1,2}$ and Susan E. Howlett ${ }^{3,4}$

\author{
Affiliations: \\ ${ }^{1}$ Department of Physiology, Anatomy, and Genetics, University of Oxford, Oxford, UK \\ ${ }^{2}$ Centre for Neural Circuits and Behaviour, University of Oxford, Oxford, UK \\ ${ }^{3}$ Department of Pharmacology, Dalhousie University, Halifax, NS, Canada \\ ${ }^{4}$ Department of Medicine (Geriatric Medicine), Dalhousie University, Halifax, NS, Canada
}


A welcome development in $21^{\text {st }}$ century urban design is the practice of daylighting, the uncovering of streams that had long been buried or directed into culverts. With an increasing focus on male-female differences in biomedical research (Maric-Bilkan et al., 2016), more light is now being shed on the physiology of sex differences. Researchers have begun to uncover key sex differences in many areas of physiology, with important implications for both males and females. In this Special Issue of Current Opinion in Physiology, we have assembled a group of international scientists who have made major contributions to our understanding of the etiology of sex differences in physiology and how these differences influence the expression of diseases.

The study of sex differences in physiology is a highly multi-disciplinary area that overlaps with other disciplines studying general physiology and neurophysiology. We asked a range of biomedical research experts studying sex differences in physiology to provide us with summaries of previous and ongoing work in their particular field, and to advocate novel research questions. In particular what are the goals of this research? Why study these differences? What physiological principles are conserved? What happens when this goes wrong? What does this mean to the health of the animal or the individual? We hope that the reviews in this volume provide a broad perspective on the field that will shape our opinions on studying sex differences in physiology in the future.

Sex differences in organ system physiology can arise from male-female differences in anatomy, variation in responses to sex steroid hormones and/or changes mediated by sex chromosomes. In this issue, Molgat-Seon et al. (2018) identify major morphological differences in the human respiratory system between the sexes, with women having smaller lungs and airways that differ in terms of shape when compared to men. These anatomical differences have important functional consequences, as women have lower pulmonary function at rest, an effect that is exacerbated by exercise when the demand on the respiratory system is high (Molgat-Seon et al., 2018). In contrast to the respiratory system, male-female differences in heart function are thought to be mediated primarily by sex steroid hormones. Here, Bernasochi et al. (2018) outline evidence that challenges the traditional view that estrogens are beneficial while androgens are detrimental in terms of the heart. They highlight the emerging role of local androgenestrogen signaling between myocardial tissues and adjacent adipose tissues in explaining sex differences in cardiac physiology and pathophysiology (Bernasochi et al., 2018). Fink and Klein (2018) argue that females develop higher antibody responses than males across the life course, although these differences are greatest during the reproductive years. The evolutionary advantage of higher humoral immunity in females may be the capacity to transmit antibodies across the placenta; this may also account for sexspecific differences in responses to vaccines at all ages (Fink \& Klein, 2018).

It is clear that sex differences in physiology are detectable not just after puberty, but can occur across the life course. In this issue, the paper by Millington and Rideout, highlights novel sex differences in development in the Drosophila model (Millington \& Rideout, 2018). There are obvious male-female differences in longevity, with women living longer than men, a pattern that is also seen in many female 
mammals (Austad \& Fischer, 2016). Cheng and Nelson (2018) argue that the highly conserved female survival advantage arises from underlying differences in physiology that protect females from many causes of death. The newly developed "Four Core Genotype" mouse models (Burgoyne \& Arnold, 2016), which allow effects of sex steroid hormones to be investigated separately from those of sex chromosomes, should help establish their distinct contributions to aging and longevity (Cheng \& Nelson, 2018). Several papers in this issue discuss emerging evidence for sex-specific differences in lifespan-extending interventions such as caloric restriction (Cheng \& Nelson, 2018; Kane et al., 2018). Both conclude that relatively few interventions studies have considered both sexes, so there is a need for more work in this area. Despite the female survival advantage, it has traditionally been acknowledged that women have higher levels of frailty than men at any age (Gordon et al., 2017). Here, Gordon and Hubbard (2018) discuss this "morbiditymortality" paradox. They question whether women are more or less frail than men, as even though women have more health problems they are less vulnerable to death. Newly described mouse models of frailty, in which sex differences also have been identified (Kane et al., 2018), may help shed light on the biological basis for these sex differences. In a timely review Galea and colleagues (2018) address an important issue that has been almost completely overlooked in research to date, the physiological changes during pregnancy. The authors make it clear that physiological challenges unique to women need to be taken into consideration for a better comprehension of women's physiology and disease.

Sex differences in physiology profoundly affect the brain and behaviour. Brain development and adult brain organization, function, and biochemistry differ by sex in both vertebrates and invertebrates. These sex differences in the brain are initiated through sex-determining genes and fetal hormonal programming (reviewed by Asahina, 2018; McKinsey et al., 2018). The review by Sorge and Strath (2018) provides excellent coverage and communication of both biological (sensory) and psychological components of sex differences in pain. Molina-García and Arantza Barrios (2018) discuss recent work highlighting sex-specific differences in learning abilities amongst animals, examining the question of how sex affects learning from the relevant ecology to the neuronal circuits underlying these differences.

The articles in this volume illustrate our progress in understanding sex differences in physiology, which has been enabled by advances in integrative physiology, molecular genetic tools, and model systems. This is an exciting and rewarding time to be researching sex-differences in physiology and we hope these papers will stimulate others to sign up to this pursuit. We trust these reviews will enlighten readers by understanding how sex differences influence physiology, behaviour, and disease. From a healthcare perspective, we hope this will provide critical knowledge on sex differences for better understanding of prevention and treatment of specific diseases. By way of example it has been recently recognized that more research is needed into sex differences in Alzheimer's disease to improve prevention, diagnosis, and treatment for both women and men (Mazure and Swendsen, 2016).

The articles published in this Special Issue showcase recent advances in our understanding of the physiology of sex differences across a wide range of systems. This work not only gives us new sex-specific 
insights into normal physiology, but may help explain why men and women are susceptible to different diseases. Daylighting sex differences in physiology gives us new information about biological variables that is relevant for both halves of the population. These differences have been there all along, but covered by convention and the belief (now recognized as largely baseless (Prendergast et al., 2014)) that female models are more variable due to the estrous cycle. Bringing them into the daylight takes some effort, but the results are revealing.

\section{REFERENCES}

Asahina, K. (2018) Sex differences in Drosophila behavior: Qualitative and Quantitative Dimorphism. Curr Opin Physio. in press.

Austad SN, Fischer KE. Sex differences in lifespan. Cell Metab. 2016;23(6):1022-1033.

Bernasochi, Bell \& Delbridge. The myocardium and sex steroid hormone influences. Curr Opin Physiol. 2018; in press.

Burgoyne PS, Arnold AP. A primer on the use of mouse models for identifying direct sex chromosome effects that cause sex differences in non-gonadal tissues. Biol Sex Differ. 2016;7:68.

Cheng, CC, and Nelson, J. Physiological basis for sex-specific differences in longevity. Curr Opin Physiol. 2018; in press.

Fink \& Klein. The evolution of greater humoral immunity in females than males: implications for vaccine efficacy. Curr Opin Physiol. 2018; in press.

Galea, L.A.M., Qiu, W., and Duarte-Guterman, P. (2018) Beyond Sex Differences: Short and Long-Term Implications of Motherhood on Women's Health. Curr Opin Physio. in press.

Gordon \& Hubbard. Physiological basis for sex-differences in frailty. Curr Opin Physiol. 2018; in press.

Gordon EH, Peel NM, Samanta M, Theou O, Howlett SE, Hubbard RE. Sex differences in frailty: A systematic review and meta-analysis. Exp Gerontol. 2017;89:30-40.

Kane, Sinclair, Mitchell \& Sarah Mitchell. Sex differences in the response to dietary restriction in rodents. Curr Opin Physiol. 2018; in press.

Kane AE, Keller KM, Heinze-Milne S, Grandy SA, Howlett SE. A murine frailty index based on clinical and laboratory measurements: links between frailty and pro-inflammatory cytokines differ in a sex-specific manner. J Gerontol A Biol Sci Med Sci. 2018; [Epub ahead of print].

Maric-Bilkan C, Arnold AP, Taylor DA, Dwinell M, Howlett SE, Wenger N, Reckelhoff JF, Sandberg K, Churchill G, Levin E, Lundberg MS. Report of the National Heart, Lung, and Blood Institute Working Group 
on Sex Differences Research in Cardiovascular Disease: Scientific Questions and Challenges. Hypertension. 2016;67(5):802-7.

Mazure, C.M., and Swendsen, J. (2016) Sex differences in Alzheimer's disease and other dementias. Lancet Neurol 5, 451-452.

McKinsey, G., Ahmed, O.M., and Shah, N.M. (2018) Neural control of sexually dimorphic social behaviors. Curr Opin Physio. in press.

Millington, J.W., and Rideout, E.J. (2018) Sex differences in Drosophila development and physiology. Curr Opin Physio. in press.

Molina-García, L., and Barrios, A. (2018) Sex differences in learning - shared principles across taxa. Curr Opin Physio. in press.

Molgat-Seon, Peters \& Sheel. Sex-differences in the human respiratory system and their impact on resting pulmonary function and the integrative response to exercise. Curr Opin Physiol. 2018; in press.

Prendergast BJ, Onishi KG, Zucker I. Female mice liberated for inclusion in neuroscience and biomedical research. Neurosci Biobehav Rev. 2014;40:1-5.

Sorge, R.E., and Strath, L.J. (2018) Sex Differences in Pain Responses. Curr Opin Physio. in press. 